\title{
Prime Radicals in Ternary Semigroups
}

\author{
Y. Sarala ${ }^{1}$, A.Anjaneyulu ${ }^{2}$ D.Madhusudhana Rao ${ }^{3}$ \\ ${ }^{1}$ Dept. of Mathematics, Nagarjuna University, Guntur, A.P. India. \\ ${ }^{2,3}$ Dept. of Mathematics, VS R \& N V R College, Tenali, A.P. India.
}

\begin{abstract}
In this paper the terms completely prime ideal, prime ideal, $m$-system. globally idempotent, semi simple elements of a ternary semigroup are Introduced. It is proved that an ideal A of a ternary semigroup $T$ is completely prime if and only if $T \backslash A$ is either sub semigroup of $T$ or empty. It is proved that if $T$ is a globally idempotent ternary semigroup then every maximal ideal of $T$ is a prime ideal of $T$. In this paper the terms completely semiprime ideal, semiprime ideal, $n$-system, $d$-system and $i$-system are introduced. It is proved that the non-empty intersection of any family of a completely prime ideal and prime ideal of a ternary semigroup $T$ is a completely semiprime ideal of $T$. It is also proved that an ideal $A$ of a ternary semigroup $T$ is completely semiprime if and only if $T \backslash A$ is a d-system of $T$ or empty. It is proved that if $N$ is an $n$-system in a ternary semigroup $T$ and $a \in N$, then there exist an $m$-system $M$ in $T$ such that $a \in M$ and $M \subseteq N$. The terms radical, complete radical of a ternary semigroup are introduced. It is proved that if $A$ and $B$ are any two ideals of a ternary semigroup $T$, then i) $A \subseteq B \Rightarrow \sqrt{A} \subseteq \sqrt{B}$ ii) $\sqrt{A B C}=\sqrt{A \cap B \cap C}=\sqrt{A} \cap \sqrt{B} \cap \sqrt{C}$ iii) $\sqrt{\sqrt{A}}=\sqrt{A}$. It is also proved that if $A$ is an ideal of ternary semigroup $T$ then $\sqrt{A}=$ $\{x \in T: M(x) \cap A \neq \varnothing\}$.

Mathematics Subject Classification (2010) : 20N12, 20N05, 20N10, $20 N 12$

Keywords: Ideal completely prime ideal, prime ideal, m-system, $n$-system, $d$-system, $i$-system, globally idempotent, semisimple elements, completely semiprime ideal, semi prime ideal, irreducible ideal, strongly irreducible ideal, radical, complete radical of ternary semigroup.
\end{abstract}

\section{Introduction}

The theory of ternary algebraic system was introduced by Lehmer [13] in 1932, but earlier such structures were studied by Kasner [10] who gave the idea of n-ary algebras . Ternary semigroups are universal algebras with one associative ternary operation. Anjaneyulu.A [1],[2] initiated the study of ideals in semigroups. S.Kar and B.K.Maity [9] initiated the study of some ideals of ternary semigroups. Sioson. F. M [18] studied about Ideal theory in ternary semigroups. Iampan . A.[7] gave the idea of Lateral ideals of ternary semigroups.

\section{Preliminaries}

DEFINITION 2.1 : Let $\mathrm{T}$ be a non-empty set. Then $\mathrm{T}$ is said to be a ternary semigroup if there exist a mapping from $\mathrm{T} \times \mathrm{T} \times \mathrm{T}$ to $\mathrm{T}$ which maps $\left(x_{1}, x_{2}, x_{3}\right) \rightarrow\left[x_{1} x_{2} x_{3}\right]$ satisfying the condition $:\left[\left[x_{1} x_{2} x_{3}\right] x_{4} x_{5}\right]=\left[x_{1}\left[x_{2} x_{3} x_{4}\right] x_{5}\right]=\left[x_{1} x_{2}\left[x_{3} x_{4} x_{5}\right]\right] \forall x_{i} \in \mathrm{T}, 1 \leq i \leq 5$.

DEFINITION 2.2 : A ternary semigroup T is said to be commutative provided for all $a, b, c \in \mathrm{T}$, we have $a b c=$ $b c a=c a b=b a c=c b a=a c b$.

DEFINITION 2.3 : An element $a$ of ternary semigroup T is said to be left identity of T provided $a a t=t$ for all $t \in \mathrm{T}$.

NOTE 2.4 : Left identity element $a$ of a ternary semigroup $\mathrm{T}$ is also called as left unital element.

DEFINITION 2.5 : An element $a$ of a ternary semigroup T is said to be a lateral identity of T provided ata $=t$ for all $t \in \mathrm{T}$.

NOTE 2.6 : Lateral identity element $a$ of a ternary semigroup $T$ is also called as lateral unital element.

DEFINITION 2.7 : An element $a$ of a ternary semigroup $\mathrm{T}$ is said to be a right identity of $\mathrm{T}$ provided taa $=t \forall t \in \mathrm{T}$.

NOTE 2.8 : Right identity element $a$ of a ternary semigroup $T$ is also called as right unital element.

DEFINITION 2.9 : An element $a$ of a ternary semigroup $\mathrm{T}$ is said to be a two sided identity of $\mathrm{T}$ provided aat $=t a a=t \forall t \in \mathrm{T}$.

NOTE 2.10 : Two-sided identity element of a ternary semigroup $\mathrm{T}$ is also called as bi-unital element. 
DEFINITION 2.11 : An element $a$ of a ternary semigroup $\mathrm{T}$ is said to be an identity provided aat $=t a a=a t a=t \forall t \in \mathrm{T}$.

NOTE 2.12: An identity element of a ternary semigroup $\mathrm{T}$ is also called as unital element.

NOTE 2.13 : An element $a$ of a ternary semigroup $\mathrm{T}$ is said to be an identity of $\mathrm{T}$ then $a$ is left identity, lateral identity and right identity of $\mathrm{T}$.

NOTATION 2.14 : Let $\mathrm{T}$ be a ternary semigroup. If $\mathrm{T}$ has an identity, let $T^{1}=\mathrm{T}$ and if $\mathrm{T}$ does not have an identity, let $T^{1}$ be the ternary semigroup $\mathrm{T}$ with an identity adjoined usually denoted by the symbol 1 .

DEFINITION 2.15 : Let $\mathrm{T}$ be ternary semigroup. A non empty subset $\mathrm{S}$ of $\mathrm{T}$ is said to be a ternary subsemigroup of $\mathrm{T}$ if $a b c \in \mathrm{S}$ for all $a, b, c \in \mathrm{S}$.

NOTE 2.16 : A non empty subset $S$ of a ternary semigroup $T$ is a ternary subsemigroup if and only if SSS $\subseteq \mathrm{S}$. DEFINITION 2.17 : Let $\mathrm{T}$ be a nonempty set. A nonempty finite sequence $a_{1}, a_{2}, \ldots a_{2 \mathrm{n}-1}$ usually written by juxtaposition $a_{1} a_{2} \ldots \ldots . a_{2 n-1}$ of elements of $\mathrm{T}$ is called word over the alphabet $\mathrm{T}$. The set $\mathrm{T}$ of all words with the operation of juxtaposition $\left(a_{1} a_{2} \ldots . . a_{2 p-1}\right)\left(b_{1} b_{2} \ldots . . b_{2 q-1}\right)\left(c_{1} c_{2} \ldots . c_{2 r-1}\right)=a_{1} a_{2} \ldots . . a_{2 p-1}$

$b_{1} b_{2} \ldots . . b_{2 q-1} c_{1} c_{2} \ldots . . c_{2 r-1}$ is a ternary semigroup called the free ternary semigroup over the alphabet $\mathrm{T}$.

DEFINITION 2.18 : A nonempty subset A of a ternary semigroup $\mathrm{T}$ is said to be left ideal of $\mathrm{T}$ if $b, c \in \mathrm{T}, a$ $\in$ A implies $b c a \in$ A.

NOTE 2.19 : A nonempty subset $A$ of a ternary semigroup $T$ is said to be a left ideal of $T$ if and only if TTA $\subseteq \mathrm{A}$.

DEFINITION 2.20 : A nonempty subset of a ternary semigroup $\mathrm{T}$ is said to be a lateral ideal of $\mathrm{T}$ if $b, c \in \mathrm{T}, a \in \mathrm{A}$ implies $b a c \in \mathrm{A}$.

NOTE 2.21 : A nonempty subset of $A$ of a ternary semigroup $T$ is a lateral ideal of $T$ if and only if $\mathrm{TAT} \subseteq \mathrm{A}$.

DEFINITION 2.22 : A nonempty subset $\mathrm{A}$ of a ternary semigroup $\mathrm{T}$ is a right ideal of $\mathrm{T}$ if $b, c \in \mathrm{T}$, $a \in \mathrm{A}$ implies $a b c \in \mathrm{A}$

NOTE 2.23 : A nonempty subset A of a ternary semigroup $\mathrm{T}$ is a right ideal of $\mathrm{T}$ if and only if ATT $\subseteq$ A.

DEFINITION 2.24 : A non-empty subset A of a ternary semigroup $\mathrm{T}$ is said to be ternary ideal or simply an ideal of $\mathrm{T}$ if $b, c \in \mathrm{T}, a \in \mathrm{A}$ implies bca $\in \mathrm{A}, b a c \in \mathrm{A}, a b c \in \mathrm{A}$.

NOTE 2.25 : A nonempty subset $A$ of a ternary semigroup $T$ is an ideal of $T$ if and only if it is left ideal, lateral ideal and right ideal of $\mathrm{T}$.

DEFINITION 2.26 : An ideal A of a ternary semigroup $\mathrm{T}$ is said to be a proper ideal of $\mathrm{T}$ if $\mathrm{A}$ is different from $\mathrm{T}$.

DEFINITION 2.27 : An ideal A of a ternary semigroup T is said to be $\boldsymbol{a}$ principal ideal provided A is an ideal generated by $\{a\}$ for some $a \in \mathrm{T}$. It is denoted by $\mathrm{J}(a)$ (or) $\langle a\rangle$.

DEFINITION 2.28 : An ideal A of a ternary semigroup $\mathrm{T}$ is said to be a maximal left ideal provided $\mathrm{A}$ is a proper left ideal of $\mathrm{T}$ and is not properly contained in any proper left ideal of $\mathrm{T}$.

DEFINITION 2.29 : An ideal A of a ternary semigroup $\mathrm{T}$ is said to be a maximal lateral ideal provided $\mathrm{A}$ is a proper lateral ideal of $\mathrm{T}$ and is not properly contained in any proper lateral ideal of $\mathrm{T}$.

DEFINITION 2.30 : An ideal A of a ternary semigroup T is said to be a maximal right ideal provided A is a proper right ideal of $\mathrm{T}$ and is not properly contained in any proper right ideal of $\mathrm{T}$.

DEFINITION 2.31 : An ideal A of a ternary semigroup T is said to be a maximal two sided ideal provided A is a proper two sided ideal of $\mathrm{T}$ and is not properly contained in any proper two sided ideal of $\mathrm{T}$.

DEFINITION 2.32 : An ideal A of a ternary semigroup T is said to be a maximal ideal provided A is a proper ideal of $\mathrm{T}$ and is not properly contained in any proper ideal of $\mathrm{T}$.

DEFINITION 2.33 : A left ideal A of a ternary semigroup $\mathrm{T}$ is said to be the principal left ideal generated by $\boldsymbol{a}$ if $\mathrm{A}$ is a left ideal generated by $\{a\}$ for some $\mathrm{a} \in \mathrm{T}$. It is denoted by $\mathrm{L}(a)$ or $\langle a\rangle_{l}$.

THEOREM 2.34 : If $\mathrm{T}$ is a ternary semigroup and $a \in \mathrm{T}$ then $\mathrm{L}(a)=a \bigcup \mathrm{TT} a$.

NOTE 2.35 : if $\mathrm{T}$ is ternary semigroup and $a \in \mathrm{T}$ then $\mathrm{L}(\mathrm{a})=T^{1} T^{1} a$.

DEFINITION 2.36 : A lateral ideal $\mathrm{A}$ of a ternary semigroup $\mathrm{T}$ is said to be the principal lateral ideal generated by $\boldsymbol{a}$ if A is a lateral ideal generated by $\{a\}$ for some $a \in \mathrm{T}$. It is denoted by $\mathrm{M}$ (a) (or) $\langle a\rangle_{m}$.

THEOREM 2.37 : If $\mathrm{T}$ is a ternary semigroup and $a \in \mathrm{T}$ then $\mathrm{M}(a)=a \bigcup \mathrm{T} a \mathrm{~T} \bigcup \mathrm{TT} a \mathrm{TT}$.

DEFINITION 2.38 : A right ideal A of a ternary semigroup $\mathrm{T}$ is said to be a principal right ideal generated by $a$ if A is a right ideal generated by $\{a\}$ for some $a \in \mathrm{T}$. It is denoted by $\mathrm{R}(a)$ (or) $\langle a\rangle_{r}$. 
THEOREM 2.39 : If $T$ is a ternary semigroup and $a \in T$ then $R(a)=a \bigcup a T T$.

NOTE $2.40:$ If $\mathrm{T}$ is a ternary semigroup and $a \in \mathrm{T}$ then $\mathrm{R}(a)=\mathrm{a} T^{1} T^{1}$

DEFINITION 2.41 : A two sided ideal A of a ternary semigroup $\mathrm{T}$ is said to be the principal two sided ideal provided $\mathrm{A}$ is a two sided ideal generated by $\{a\}$ for some

$a \in \mathrm{T}$. It is denoted by $\mathrm{T}(a)$ (or) $\langle a\rangle_{t}$.

THEOREM $2.42:$ If $\mathrm{T}$ is a ternary semigroup and $a \in \mathrm{T}$ then $\mathrm{T}(a)=a \bigcup \mathrm{TT} a \bigcup a \mathrm{TT} \bigcup \mathrm{TT} a \mathrm{TT}$.

DEFINITION 2.43 : An ideal A of a ternary semigroup $\mathrm{T}$ is said to be $\boldsymbol{a}$ principal ideal provided $\mathrm{A}$ is an ideal generated by $\{a\}$ for some $a \in \mathrm{T}$. It is denoted by $\mathrm{J}$ (a) (or) $\langle a\rangle$.

THEOREM $2.44:$ If $\mathrm{T}$ is a ternary semigroup and $\mathrm{a} \in \mathrm{T}$ then

$\mathrm{J}(a)=a \bigcup a \mathrm{TT} \bigcup \mathrm{TT} a \bigcup \mathrm{T} a \mathrm{~T} \bigcup \mathrm{TT} a \mathrm{TT}$.

NOTE 2.45: If $\mathrm{T}$ is a ternary semigroup and $\mathrm{a} \in \mathrm{T}$ then

$\mathrm{J}(a)=a \bigcup a \mathrm{TT} \bigcup \mathrm{TT} a \bigcup \mathrm{T} a \mathrm{~T} \bigcup \mathrm{TT} a \mathrm{TT}=T^{1} T^{1} a T^{1} T^{1}$.

\section{Completely Prime Ideals And Prime Ideals}

DEFINITION 3.1 : An ideal A of a ternary semigroup T is said to be a completely prime ideal of T provided $x$, $y, z \in \mathrm{T}$ and $x y z \in \mathrm{A}$ implies either $x \in \mathrm{A}$ or $y \in \mathrm{A}$ or $z \in \mathrm{A}$.

EXAMPLE 3.2 $:$ In the commutative ternary semigroup $Z^{-}$of all negative integers, the ideal $P=\{3 \mathrm{k}: \mathrm{k} \in$ $Z^{-}$\} is a completely prime ideal. For $x ; y ; z \in Z^{-}$, xyz $\in \mathrm{P} \Leftrightarrow x y z$ is divisible by $3 \Leftrightarrow x$ is divisible by 3 or $y$ is divisible by 3 or $z$ is divisible by $3 \Leftrightarrow x=3 k_{1}$ or $y=3 k_{2}$ or $z=3 k_{3}$ for $k_{1} ; k_{2} ; k_{3} \in Z^{-} \Leftrightarrow x \in P$ or $y \in P$ or $z \in P$.

EXAMPLE 3.3 : In example 3.2., $P$ is a completely prime ideal. But the ideal $Q=\left\{30 \mathrm{k}: \mathrm{k} \in Z^{-}\right\}$is not a prime ideal of $Z^{-}$, since $(-2)(-3)(-5)=-30 \in \mathrm{Q}$ but $(-2) \notin \mathrm{Q},(-3) \notin \mathrm{Q}$ and $(-5) \notin \mathrm{Q}$.

THEOREM 3.4 : An ideal $\mathrm{A}$ of a ternary semigroup $\mathrm{T}$ is completely prime if and only if $x_{1}, x_{2}, \ldots . ., x_{n} \in \mathrm{T}$, $n$ is odd natural number, $x_{1} x_{2} \ldots \ldots x_{n} \in \mathrm{A} \Rightarrow x_{i} \in \mathrm{A}$ for some $i=1,2,3, \ldots . . n$.

Proof: Suppose that A is a completely prime ideal of T.

Let $x_{1}, x_{2}, \ldots . ., x_{n} \in \mathrm{T}$ where $n$ is odd natural number and $x_{1} x_{2} \ldots . x_{n} \in \mathrm{A}$.

If $n=1$ then clearly $x_{1} \in \mathrm{A}$.

If $n=3$ then $x_{1} x_{2} x_{3} \in \mathrm{A} \Rightarrow x_{1} \in \mathrm{A}$ or $x_{2} \in \mathrm{A}$ or $x_{3} \in \mathrm{A}$.

If $n=5$ then $x_{1} x_{2} x_{3} x_{4} x_{5} \in \mathrm{A} \Rightarrow x_{1} x_{2} x_{3} \in \mathrm{A}$ or $x_{4} \in \mathrm{A}$ or $x_{5} \in \mathrm{A}$

$\Rightarrow x_{1} \in \mathrm{A}$ or $x_{2} \in \mathrm{A}$ or $x_{3} \in \mathrm{A}$ or $x_{4} \in \mathrm{A}$ or $x_{5} \in \mathrm{A}$.

Therefore by induction of $n$ is an odd natural number, then $x_{1} x_{2} \ldots . . x_{n} \in \mathrm{A}$

$\Rightarrow x_{i} \in \mathrm{A}$ for some $i=1,2,3, \ldots . . n$.

The converse part is trivial.

THEOREM 3.5 : An ideal A of a ternary semigroup $T$ is completely prime if and only if T\A is either subsemigroup of $T$ or empty.

Proof: Suppose that $\mathrm{A}$ is a completely prime ideal of $\mathrm{T}$ and $\mathrm{T} \backslash \mathrm{A} \neq \varnothing$.

Let $a, b, c \in \mathrm{T} \backslash \mathrm{A}$. Then $a \notin \mathrm{A}, b \notin \mathrm{A}, c \notin \mathrm{A}$. Suppose if possible $a b c \notin \mathrm{T} \backslash \mathrm{A}$.

Then $a b c \in \mathrm{A}$. Since A is completely prime, either $a \in \mathrm{A}$ or $b \in \mathrm{A}$ or $c \in \mathrm{A}$.

It is a contradiction. Therefore $a b c \in \mathrm{T} \backslash \mathrm{A}$. Hence $\mathrm{T} \backslash \mathrm{A}$ is a subsemigroup of $\mathrm{T}$.

Conversely suppose that $\mathrm{T} \backslash \mathrm{A}$ is a subsemigroup of $\mathrm{T}$ or $\mathrm{T} \backslash \mathrm{A}$ is empty.

If $\mathrm{T} \backslash \mathrm{A}$ is empty then $\mathrm{A}=\mathrm{T}$ and hence $\mathrm{A}$ is completely prime.

Assume that T\A is a subsemigroup of $\mathrm{T}$. Let $a, b, c \in \mathrm{T}$ and $a b c \in \mathrm{A}$.

Suppose if possible $a \notin \mathrm{A}, b \notin \mathrm{A}$, and $c \notin \mathrm{A}$.

Then $a \in \mathrm{T} \backslash \mathrm{A}, b \in \mathrm{T} \backslash \mathrm{A}$ and $c \in \mathrm{T} \backslash \mathrm{A}$. Since $\mathrm{T} \backslash \mathrm{A}$ is a subsemigroup, $a b c \in \mathrm{T} \backslash \mathrm{A}$ and hence $a b c \notin \mathrm{A}$. It is a contradiction. Hence either $a \in \mathrm{A}$ or $b \in \mathrm{A}$ or $c \in \mathrm{A}$. Therefore $\mathrm{A}$ is a completely prime ideal of $\mathrm{T}$.

DEFINITION 3.6 : An ideal A of a ternary semigroup $\mathrm{T}$ is said to be a prime ideal of $\mathrm{T}$ provided $\mathrm{X}, \mathrm{Y}, \mathrm{Z}$ are ideals of $\mathrm{T}$ and $\mathrm{XYZ} \subseteq \mathrm{A} \Rightarrow \mathrm{X} \subseteq \mathrm{A}$ or $\mathrm{Y} \subseteq \mathrm{A}$ or $\mathrm{Z} \subseteq \mathrm{A}$.

THEOREM 3.7 : In a ternary semigroup $T$, the following conditions are equivalent:

(i) $\mathbf{A}$ is a prime ideal of $\mathbf{T}$.

(ii) For $a, b, c \in \mathrm{T}$; $\langle a\rangle\langle b\rangle\langle c\rangle \subseteq \mathrm{A}$ implies $a \in \mathrm{A}$ or $b \in \mathrm{A}$ or $c \in \mathrm{A}$.

(iii) For $a ; b ; c \in \mathbf{T} ; \mathbf{T}^{1} \mathbf{T}^{1} a \mathbf{T}^{1} \mathbf{T}^{1} b \mathbf{T}^{1} \mathbf{T}^{1} c \mathbf{T}^{1} \mathbf{T}^{1} \subseteq \mathrm{A}$ implies $a \in A$ or $b \in \mathrm{A}$ or $c \in A$.

Proof: (i) $\Rightarrow$ (ii): Suppose that A is a prime ideal of T. Then (i) $\Rightarrow$ (ii) is obvious.

(ii) $\Rightarrow$ (iii): Let $a, b, c \in \mathrm{T}$ such that $\mathrm{T}^{1} \mathrm{~T}^{1} a \mathrm{~T}^{1} \mathrm{~T}^{1} b \mathrm{~T}^{1} \mathrm{~T}^{1} c \mathrm{~T}^{1} \mathrm{~T}^{1} \subseteq \mathrm{A}$. 
Now $\langle a\rangle\langle b\rangle\langle c\rangle=\left(\mathrm{T}^{1} \mathrm{~T}^{1} a \mathrm{~T}^{1} \mathrm{~T}^{1}\right)\left(\mathrm{T}^{1} \mathrm{~T}^{1} b \mathrm{~T}^{1} \mathrm{~T}^{1}\right)\left(\mathrm{T}^{1} \mathrm{~T}^{1} c \mathrm{~T}^{1} \mathrm{~T}^{1}\right) \subseteq \mathrm{T}^{1} \mathrm{~T}^{1} a \mathrm{~T}^{1} \mathrm{~T}^{1} b \mathrm{~T}^{1} \mathrm{~T}^{1} c \mathrm{~T}^{1} \mathrm{~T}^{1} \subseteq \mathrm{A}$ $\Rightarrow a \in \mathrm{A}$ or $b \in \mathrm{A}$ or $c \in \mathrm{A}$.

(iii) $\Rightarrow$ (i): Suppose that $a, b, c \in \mathrm{T} ; \mathrm{T}^{1} \mathrm{~T}^{1} a \mathrm{~T}^{1} \mathrm{~T}^{1} b \mathrm{~T}^{1} \mathrm{~T}^{1} c \mathrm{~T}^{1} \mathrm{~T}^{1} \subseteq \mathrm{A} \Rightarrow a \in \mathrm{A}$ or $b \in \mathrm{A}$ or $c \in \mathrm{A}$.

Let $\mathrm{X}, \mathrm{Y}, \mathrm{Z}$ be the three ideals of $\mathrm{T}$ and $\mathrm{XYZ} \subseteq \mathrm{A}$.

Suppose if possible $\mathrm{X} \nsubseteq \mathrm{A}, \mathrm{Y} \nsubseteq \mathrm{A}, \mathrm{Z} \nsubseteq \mathrm{A}$.

$\mathrm{X} \nsubseteq \mathrm{A}, \mathrm{Y} \nsubseteq \mathrm{A}, \mathrm{Z} \nsubseteq \mathrm{A}$, there exists $a, b, c$ such that $a \in \mathrm{X}$ and $a \notin \mathrm{A}, b \in \mathrm{Y}$ and $b \notin \mathrm{A}$ and $c \in \mathrm{Z}$ and $c \notin \mathrm{A} . a$ $\in \mathrm{X}, b \in \mathrm{Y}, c \in \mathrm{Z} \Rightarrow a b c \in \mathrm{XYZ} \subseteq \mathrm{A}$.

Now $\mathrm{T}^{1} \mathrm{~T}^{1} a \mathrm{~T}^{1} \mathrm{~T}^{1} b \mathrm{~T}^{1} \mathrm{~T}^{1} c \mathrm{~T}^{1} \mathrm{~T}^{1} \subseteq \mathrm{XYZ} \subseteq \mathrm{A} \Rightarrow a \in \mathrm{A}$ or $b \in \mathrm{A}$ or $c \in \mathrm{A}$. It is a contradiction.

Therefore $\mathrm{X} \subseteq \mathrm{A}$ or $\mathrm{Y} \subseteq \mathrm{A}$ or $\mathrm{Z} \subseteq \mathrm{A}$ and hence $\mathrm{A}$ is a prime ideal of $\mathrm{T}$.

THEOREM 3.8 : An ideal A of a ternary semigroup $\mathrm{T}$ is prime if and only if $X_{1}, X_{2}, \ldots . ., X_{n} \subseteq \mathrm{T}, n$ is odd natural number, $X_{1} X_{2} \ldots . . \mathrm{X}_{n} \subseteq \mathrm{A} \Rightarrow X_{i} \in \mathrm{A}$ for some $i=1,2,3, \ldots . . n$.

Proof: Suppose that $\mathrm{A}$ is a prime ideal of T.

Let $X_{1}, X_{2}, \ldots . ., X_{n} \subseteq \mathrm{T}, n$ is odd natural number and $X_{1} X_{2} \ldots \ldots \mathrm{X}_{n} \subseteq \mathrm{A}$

If $n=1$ then clearly $X_{1} \in \mathrm{A}$.

If $n=3$ then $X_{1} X_{2} X_{3} \subseteq \mathrm{A} \Rightarrow X_{1} \subseteq \mathrm{A}$ or $X_{2} \subseteq \mathrm{A}$ or $X_{3} \subseteq \mathrm{A}$.

If $n=5$ then $X_{1} X_{2} X_{3} X_{4} X_{5} \subseteq \mathrm{A} \Rightarrow X_{1} X_{2} X_{3} \in \mathrm{A}$ or $X_{4} \in \mathrm{A}$ or $X_{5} \in \mathrm{A}$

$\Rightarrow X_{1} \in \mathrm{A}$ or $X_{2} \in \mathrm{A}$ or $X_{3} \in \mathrm{A}$ or $X_{4} \in \mathrm{A}$ or $X_{5} \in \mathrm{A}$.

Therefore by induction of $n$ is an odd natural number, then $X_{1} X_{2} \ldots \ldots \mathrm{X}_{n} \subseteq \mathrm{A}$

$\Rightarrow X_{i} \subseteq$ A for some $i=1,2,3, \ldots . . n$.

The converse part is trivial.

THEOREM 3.9 : Every completely prime ideal of a ternary semigroup $\mathbf{T}$ is a prime ideal of $T$.

Proof: Suppose that A is a completely prime ideal of a ternary semigroup T.

Let $a, b, c \in \mathrm{T}$ and $\langle a\rangle\langle b\rangle\langle c\rangle \subseteq \mathrm{A}$. Then $a b c \in \mathrm{A}$. Since A is a completely prime, either $a \in \mathrm{A}$ or $b \in \mathrm{A}$ or $c \in \mathrm{A}$. Therefore $\mathrm{A}$ is a prime ideal of $\mathrm{T}$.

The following theorem is duo to Kar.S and Maity.B.K. [9].

THEOREM 3.10 : Let $T$ be a commutative ternary semigroup . An ideal $P$ of $T$ is a prime ideal if and only if $P$ is a completely prime ideal.

DEFINITION 3.11 : A nonempty subset $A$ of a ternary semigroup $T$ is said to be an $\boldsymbol{m}$-system provided for any $a, b, c \in \mathrm{A}$ implies that $\mathrm{T}^{1} \mathrm{~T}^{1} a \mathrm{~T}^{1} \mathrm{~T}^{1} b \mathrm{~T}^{1} \mathrm{~T}^{1} c \mathrm{~T}^{1} \mathrm{~T}^{1} \cap \mathrm{A} \neq \emptyset$.

THEOREM 3.12 : An ideal $A$ of a ternary semigroup $T$ is a prime ideal of $T$ if and only if $T \backslash A$ is an $m$ system of $T$ or empty.

Proof : Suppose that $\mathrm{A}$ is a prime ideal of a ternary semigroup $\mathrm{T}$ and $\mathrm{T} \backslash \mathrm{A} \neq \varnothing$.

Let $a, b, c \in \mathrm{T} \backslash \mathrm{A}$. Then $a \notin \mathrm{A}, b \notin \mathrm{A}$ and $c \notin \mathrm{A}$.

Suppose if possible $\mathrm{T}^{1} \mathrm{~T}^{1} a \mathrm{~T}^{1} \mathrm{~T}^{1} b \mathrm{~T}^{1} \mathrm{~T}^{1} c \mathrm{~T}^{1} \mathrm{~T}^{1} \cap \mathrm{T} \backslash \mathrm{A}=\varnothing$.

$\mathrm{T}^{1} \mathrm{~T}^{1} a \mathrm{~T}^{1} \mathrm{~T}^{1} b \mathrm{~T}^{1} \mathrm{~T}^{1} c \mathrm{~T}^{1} \mathrm{~T}^{1} \cap \mathrm{T} \backslash \mathrm{A}=\emptyset \Rightarrow \mathrm{T}^{1} \mathrm{~T}^{1} a \mathrm{~T}^{1} \mathrm{~T}^{1} b \mathrm{~T}^{1} \mathrm{~T}^{1} c \mathrm{~T}^{1} \mathrm{~T}^{1} \subseteq \mathrm{A}$.

Since $\mathrm{A}$ is prime, either $a \in \mathrm{A}$ or $b \in \mathrm{A}$ or $c \in \mathrm{A}$.

It is a contradiction. Therefore $\mathrm{T}^{1} \mathrm{~T}^{1} a \mathrm{~T}^{1} \mathrm{~T}^{1} b \mathrm{~T}^{1} \mathrm{~T}^{1} c \mathrm{~T}^{1} \mathrm{~T}^{1} \cap \mathrm{T} \backslash \mathrm{A} \neq \emptyset$.

Hence $\mathrm{T} \backslash \mathrm{A}$ is an $m$-system.

Conversely suppose that $\mathrm{T} \backslash \mathrm{A}$ is either an $m$-system of $\mathrm{T}$ or $\mathrm{T} \backslash \mathrm{A}=\varnothing$.

If $\mathrm{T} \backslash \mathrm{A}=\varnothing$, then $\mathrm{T}=\mathrm{A}$ and hence $\mathrm{A}$ is a prime ideal of $\mathrm{T}$.

Assume that $\mathrm{T} \backslash \mathrm{A}$ is an $m$-system of T. Let $a, b, c \in \mathrm{T}$ and $\langle a\rangle\langle b\rangle\langle c\rangle \subseteq \mathrm{A}$.

Suppose if possible $a \notin \mathrm{A}, b \notin \mathrm{A}$ and $c \notin \mathrm{A}$. Then $a, b, c \in \mathrm{T} \backslash \mathrm{A}$. Sine $\mathrm{T} \backslash \mathrm{A}$ is an $m$-system,

$\Rightarrow \mathrm{T}^{1} \mathrm{~T}^{1} a \mathrm{~T}^{1} \mathrm{~T}^{1} b \mathrm{~T}^{1} \mathrm{~T}^{1} c \mathrm{~T}^{1} \mathrm{~T}^{1} \cap \mathrm{T} \backslash \mathrm{A} \neq \varnothing \Rightarrow \mathrm{T}^{1} \mathrm{~T}^{1} a \mathrm{~T}^{1} \mathrm{~T}^{1} b \mathrm{~T}^{1} \mathrm{~T}^{1} c \mathrm{~T}^{1} \mathrm{~T}^{1} \nsubseteq \mathrm{A}$

$\Rightarrow\langle a\rangle\langle b\rangle\langle c\rangle \nsubseteq \mathrm{A}$. It is a contradiction.

Therefore $a \in \mathrm{A}$ or $b \in \mathrm{A}$ or $c \in \mathrm{A}$. Hence $\mathrm{A}$ is a prime ideal of $\mathrm{T}$.

DEFINITION 3. 13 : An ideal $\mathrm{A}$ of a ternary semigroup $\mathrm{T}$ is called a globally idempotent ideal if $\mathrm{A}^{n}=\mathrm{A}$ for all odd natural number $n$.

DEFINITION 3.14 : A ternary semigroup $\mathrm{T}$ is said to be a globally idempotent ternary semigroup if $\mathrm{T}^{n}=\mathrm{T}$ for all odd natural number $n$.

THEOREM 3.15 : If $T$ is a globally idempotent ternary semigroup then every maximal ideal of $T$ is a prime ideal of $T$.

Proof : Let M be a maximal ideal of T. Let A, B, C be three ideals of T such that

$\mathrm{ABC} \subseteq \mathrm{M}$. Suppose if possible $\mathrm{A} \nsubseteq \mathrm{M}, \mathrm{B} \nsubseteq \mathrm{M}, \mathrm{C} \nsubseteq \mathrm{M}$.

Now $\mathrm{A} \nsubseteq \mathrm{M} \Rightarrow \mathrm{M} \bigcup \mathrm{A}$ is an ideal of $\mathrm{T}$ and $\mathrm{M} \subset \mathrm{M} \bigcup \mathrm{A} \subseteq \mathrm{T}$.

Since $M$ is a maximal, $M \cup A=T$.

Similarly $\mathrm{B} \nsubseteq \mathrm{M} \Rightarrow \mathrm{M} \cup \mathrm{B}=\mathrm{T}, \mathrm{C} \nsubseteq \mathrm{M} \Rightarrow \mathrm{M} \cup \mathrm{C}=\mathrm{T}$. 
Now $\mathrm{T}=\mathrm{TTT}=(\mathrm{M} \cup \mathrm{A})(\mathrm{M} \cup \mathrm{B})(\mathrm{M} \cup \mathrm{C}) \subseteq \mathrm{M} \Rightarrow \mathrm{T} \subseteq \mathrm{M}$. Thus $\mathrm{M}=\mathrm{T}$.

It is a contradiction. Therefore either $\mathrm{A} \subseteq \mathrm{M}$ or $\mathrm{B} \subseteq \mathrm{M}$ or $\mathrm{C} \subseteq \mathrm{M}$. Hence $\mathrm{M}$ is a prime.

DEFINITION 3.16 : An element $a$ of a ternary semigroup T is said to be semisimple if $n$ is odd natural number then $a \in\langle a\rangle^{n}$ i.e. $\langle a\rangle^{n}=\langle a\rangle$.

DEFINITION 3.17 : A ternary semigroup $T$ is called semisimple ternary semigroup provided every element in $\mathrm{T}$ is semisimple.

THEOREM 3.18 : If $\mathrm{T}$ is a globally idempotent ternary semigroup having maximal ideals then $\mathrm{T}$ contains semisimple elements.

Proof: Suppose that $\mathrm{T}$ is a globally idempotent ternary semigroup having maximal ideals.

Let $\mathrm{M}$ be a maximal ideal of $\mathrm{T}$. Then by theorem 3.15., $\mathrm{M}$ is prime.

Now if $a \in \mathrm{T} \backslash \mathrm{M}$ then $\langle a\rangle \nsubseteq \mathrm{M}$ and $\langle a\rangle^{\mathrm{n}} \nsubseteq \mathrm{M}$. Then $\mathrm{T}=\mathrm{M} \cup\langle a\rangle=\mathrm{M} \cup\langle a\rangle^{\mathrm{n}}$.

Therefore $a \in\langle a\rangle^{\mathrm{n}}$ and hence $\langle a\rangle=\langle a\rangle^{\mathrm{n}}$. Thus $a$ is a semisimple element. Therefore $\mathrm{T}$ contains semisimple elements.

\section{Completely Semiprime Ideals And Semiprime Ideals}

DEFINITION 4.1 : An ideal A of a ternary semigroup T is said to be a completely semiprime ideal provided $x$ $\in \mathrm{T}, x^{n} \in \mathrm{A}$ for some odd natural number $n>1$ implies $x \in \mathrm{A}$.

EXAMPLE 4.2 : In commutative ternary semigroup $\mathrm{Z}^{-}$of all negative integers, the ideal $\mathrm{Q}=\left\{6 k: k \in \mathrm{Z}^{-}\right\}$is a semiprime ideal. For $x \in \mathrm{Z}^{-}, x^{3} \in \mathrm{Q} \Leftrightarrow x^{3}$ is divisible by $6 \Leftrightarrow x$ is divisible by $6 \Leftrightarrow x=6 k_{1}$ for $k_{1} \in \mathrm{Z}^{-} \Leftrightarrow x \in$ Q.

THEOREM 4.3 : An ideal A of a ternary semigroup T is completely semiprime if and only if $x \in \mathrm{T}, x^{3} \in \mathrm{A}$ implies $x \in \mathrm{A}$.

Proof: Suppose that $\mathrm{A}$ is a completely semiprime ideal of $\mathrm{T}$.

Then clearly $x \in \mathrm{T}, x^{3} \in \mathrm{A} \Rightarrow x \in \mathrm{A}$.

Conversely suppose that $x \in \mathrm{T}, x^{3} \in \mathrm{A} \Rightarrow x \in \mathrm{A}$.

We prove that $x \in \mathrm{T}, x^{n} \in \mathrm{A}$, for some odd natural number $\mathrm{n}>1 \Rightarrow x \in \mathrm{A} \rightarrow(1)$,

by induction on $n$. Clearly (1) is true for $n=3$. Assume that (1) is true for $n=k$. i.e., $x^{k} \in A \Rightarrow x \in \mathrm{A}$ for some odd natural number $k>3$.

Suppose that $x^{k+2} \in A$. Then $x^{k+2} \in A \Rightarrow x^{k+2} \cdot x^{k+2} \cdot x^{k-4} \in A \Rightarrow x^{3 k} \in A \Rightarrow\left(x^{k}\right)^{3} \in \mathrm{A} \Rightarrow x^{k} \in A \Rightarrow x \in A$. Therefore $x^{k} \in A \Rightarrow x \in A$.

By induction, $x^{n} \in \mathrm{A}$ for some natural number $\mathrm{n}, n>1$ implies $x \in \mathrm{A}$.

Therefore A is completely semiprime.

THEOREM $4.4:$ If $\mathrm{A}$ is a completely semiprime ideal of a ternary semigroup $\mathrm{T}$, then $x, y, z \in \mathrm{T}, x y z \in \mathrm{A}$ implies that $x y \mathrm{TT} z \subseteq \mathrm{A}$ and $x \mathrm{TT} y z \subseteq \mathrm{A}$.

Proof: Let A be a completely semiprime ideal of a semigroup T. Let $x, y, z \in \mathrm{T}, x y z \in \mathrm{A}$.

Now $x y z \in \mathrm{A} \Rightarrow(z x y)^{3}=(z x y)(z x y)(z x y)=z(x y z)(x y z) x y \in \mathrm{A}$.

$(z x y)^{3} \in \mathrm{A}, \mathrm{A}$ is completely semiprime implies $z x y \in \mathrm{A}$.

Let $s, t \in \mathrm{T}$. Consider $(x y s t z)^{3}=(x y s t z)(x y s t z)(x y s t z)=x y s t(z x y) s t(z x y) s t y \in \mathrm{A}$.

$(x y s t z)^{3} \in \mathrm{A}, \mathrm{A}$ is completely semiprime implies xystz $\in \mathrm{A}$.

Therefore $x, y, z \in \mathrm{T}, x y z \in \mathrm{A} \Rightarrow x y s t z \in A$ for all $s, t \in \mathrm{T} \Rightarrow x y \mathrm{TT} z \subseteq \mathrm{A}$.

Now $x y z \in \mathrm{A} \Longrightarrow(y z x)^{3}=(y z x)(y z x)(y z x)=y z(x y z)(x y z) x \in \mathrm{A}$.

$(y z x)^{3} \in \mathrm{A}, \mathrm{A}$ is completely semiprime implies $\Rightarrow y z x \in \mathrm{A}$.

Let $s, t \in \mathrm{T}$. Consider $(x s t y z)^{3}=(x s t y z)(x s t y z)(x s t y z)=x s t(y z x) s t(y z x) s t y z \in \mathrm{A}$.

$(x s t y z)^{3} \in \mathrm{A}, \mathrm{A}$ is completely semiprime implies $x$ styz $\in \mathrm{A}$.

Therefore $x, y, z \in \mathrm{T}, x s t y z \in \mathrm{A}$ for all $s, t \in \mathrm{T} \Rightarrow x \mathrm{TT} y z \subseteq \mathrm{A}$.

COROLLARY 4.5 : If an ideal A of a ternary semigroup $\mathrm{T}$ is completely semiprime then $x, y, z \in \mathrm{T}, x y z \in$ $\mathrm{A} \Rightarrow\langle x\rangle\langle y\rangle\langle z\rangle \subseteq \mathrm{A}$.

THEOREM 4.6 : Every completely prime ideal of a ternary semigroup $\mathrm{T}$ is a completely semiprime ideal of $\mathbf{T}$.

Proof : Let A be a completely prime ideal of a ternary semigroup T. Suppose that

$x \in \mathrm{T}$ and $x^{3} \in \mathrm{A}$. Since A is a completely prime ideal of T, $x \in \mathrm{A}$.

Therefore $\mathrm{T}$ is a completely semiprime ideal.

THEOREM 4.7 : Let A be a prime ideal of a ternary semigroup T. If A is completely semiprime ideal of $T$ then $A$ is completely prime.

Proof : Let $x, y, z \in \mathrm{T}$ and $x y z \in \mathrm{A}$. Since $\mathrm{A}$ is completely semiprime, by theorem 4.4., $x y z \in \mathrm{A} \Rightarrow x y \mathrm{~T}^{1} \mathrm{~T}^{1} z \subseteq \mathrm{A}, x \mathrm{~T}^{1} \mathrm{~T}^{1} y z \subseteq \mathrm{A} \Rightarrow \mathrm{T} x y \mathrm{TT} z \mathrm{~T} \subseteq \mathrm{TAT} \subseteq \mathrm{A} \Rightarrow\langle x\rangle\langle y\rangle\langle z\rangle \subseteq \mathrm{A}$

$\Rightarrow x \in \mathrm{A}$ or $y \in \mathrm{A}$ or $z \in \mathrm{A}$ and hence $\mathrm{A}$ is completely prime. 
THEOREM 4.8 : The nonempty intersection of any family of a completely prime ideal of a ternary semigroup $T$ is a completely semiprime ideal of $T$.

Proof : Let $\left\{A_{\alpha}\right\}_{\alpha \in \Delta}$ be a family of a completely prime ideals of T such that $\bigcap_{\alpha \in \Delta} A_{\alpha} \neq \varnothing$.

It is clear that $\bigcap_{\alpha \in \Delta} A_{\alpha}$ is an ideal. Let $a \in \mathrm{T}$ and $a^{3} \in \bigcap_{\alpha \in \Delta} A_{\alpha}$. Then $a^{3} \in A_{\alpha}$ for all $\alpha \in \Delta$.

Since $A_{\alpha}$ is completely prime, $a \in A_{\alpha}$ for all $\alpha \in \Delta$ and hence $a \in \bigcap_{\alpha \in \Delta} A_{\alpha}$.

Therefore $\bigcap_{\alpha \in \Delta} A_{\alpha}$ is a completely semiprime ideal of $\mathrm{T}$.

DEFINITION 4.9 : Let T be a ternary semigroup. A non-empty subset A of T is said to be a $d$-system of $\mathrm{T}$ if $a$ $\in \mathrm{A} \Rightarrow a^{n} \in \mathrm{A}$ for all odd natural number $n$.

THEOREM 4.10 : An ideal A of a ternary semigroup T is completely semiprime if and only if T\A is a $d$ system of $T$ or empty.

Proof: Suppose that $\mathrm{A}$ is a completely semiprime ideal of $\mathrm{T}$ and $\mathrm{T} \backslash \mathrm{A} \neq \varnothing$.

Let $a \in \mathrm{T} \backslash \mathrm{A}$. Then $a \notin \mathrm{A}$. Suppose if possible $a^{n} \notin \mathrm{T} \backslash \mathrm{A}$ for some odd natural number $n$.

Then $a^{n} \in \mathrm{A}$. Since $\mathrm{A}$ is a completely semiprime ideal then $a \in \mathrm{A}$.

It is a contradiction. Therefore $a^{n} \in \mathrm{T} \backslash \mathrm{A}$ and hence $\mathrm{T} \backslash \mathrm{A}$ is a $d$-system .

Conversely suppose that $\mathrm{T} \backslash \mathrm{A}$ is a $d$-system of $\mathrm{T}$ or T\A is empty.

If $\mathrm{T} \backslash \mathrm{A}$ is empty then $\mathrm{T}=\mathrm{A}$ and hence $\mathrm{A}$ is completely semiprime.

Assume that T\A is a $d$-system of T. Let $a \in \mathrm{T}$ and $a^{n} \in \mathrm{A}$.

Suppose if possible $a \notin \mathrm{A}$. Then $a \in \mathrm{T} \backslash \mathrm{A}$.

Since T\A is a $d$-system, $a^{n} \in \mathrm{T} \backslash \mathrm{A}$. It is a contradiction. Hence $a \in \mathrm{A}$.

Thus $\mathrm{A}$ is a completely semiprime ideal of $\mathrm{T}$.

DEFINITION 4.11 : An ideal A of a ternary semigroup $\mathrm{T}$ is said to be semiprime ideal provided $\mathrm{X}$ is an ideal of $\mathrm{T}$ and $\mathrm{X}^{n} \subseteq \mathrm{A}$ for some odd natural number $n$ implies $\mathrm{X} \subseteq \mathrm{A}$.

THEOREM 4.12 : An ideal $A$ of a ternary semigroup $T$ is semiprime if and only if $X$ is an ideal of $T, X^{3} \subseteq$ A implies $\mathbf{X} \subseteq \mathrm{A}$.

Proof: Suppose that $\mathrm{A}$ is a semiprime ideal. Then clearly $\mathrm{X}^{3} \subseteq \mathrm{A} \Rightarrow \mathrm{X} \subseteq \mathrm{A}$.

Conversely suppose that $\mathrm{X}$ is an ideal of $\mathrm{T}, \mathrm{X}^{3} \subseteq \mathrm{A} \Rightarrow \mathrm{X} \subseteq \mathrm{A}$.

We prove that $\mathrm{X}^{\mathrm{n}} \subseteq \mathrm{A}$, for some odd natural number $n \Rightarrow \mathrm{X} \subseteq \mathrm{A} \rightarrow(1)$, by induction on $n$. Since $\mathrm{X}^{3} \subseteq \mathrm{A} \Rightarrow \mathrm{X}$ $\subseteq \mathrm{A},(1)$ is true for $n=3$.

Assume that $\mathrm{X}^{\mathrm{k}} \subseteq \mathrm{A}$ for some odd natural number $\mathrm{k}, 1 \leq k<n \Rightarrow \mathrm{X} \subseteq \mathrm{A}$.

Now $\mathrm{X}^{\mathrm{k}+2} \subseteq \mathrm{A} \Rightarrow \mathrm{X}^{k+2} \cdot \mathrm{X}^{k+2} \cdot \mathrm{X}^{k-4} \subseteq \mathrm{A} \Rightarrow \mathrm{X}^{3 \mathrm{k}} \subseteq \mathrm{A} \Rightarrow\left(\mathrm{X}^{\mathrm{k}}\right)^{3} \subseteq \mathrm{A} \Rightarrow \mathrm{X}^{\mathrm{k}} \subseteq \mathrm{A} \Rightarrow \mathrm{X} \subseteq \mathrm{A}$ by assumption. By induction $\mathrm{X}^{n} \subseteq \mathrm{A}$ for some odd natural number $n \Rightarrow \mathrm{X} \subseteq \mathrm{A}$.

Therefore A is semiprime.

THEOREM 4.13 : Every prime ideal of a ternary semigroup is semiprime.

Proof : Suppose that A is a prime ideal of a ternary semigroup T. Let $\mathrm{X}$ be an ideal of $\mathrm{T}$ such that $\mathrm{X}^{3} \subseteq \mathrm{A}$. Since $\mathrm{A}$ is prime, $\mathrm{X} \subseteq \mathrm{A}$. Hence $\mathrm{A}$ is semiprime.

THEOREM 4.14 : If $A$ is an ideal of a ternary semigroup $T$ then the following are equivalent.

1. A is a semiprime ideal.

2. For $a \in \mathrm{T} ;\langle a\rangle^{3} \subseteq \mathrm{A}$ implies $a \in \mathrm{A}$.

3. For $a \in \mathbf{T} ; \mathbf{T}^{1} \mathbf{T}^{1} a \mathbf{T}^{1} \mathbf{T}^{1} a \mathbf{T}^{1} \mathbf{T}^{1} a \mathbf{T}^{1} \mathbf{T}^{1} \subseteq \mathrm{A}$ implies $a \in \mathbf{A}$.

Proof: (i) $\Rightarrow$ (ii) : Suppose that A is a semiprime ideal of T. Then (i) $\Rightarrow$ (ii) is obvious.

(ii) $\Rightarrow$ (iii): Let $a \in \mathrm{T}$ such that $\mathrm{T}^{1} \mathrm{~T}^{1} a \mathrm{~T}^{1} \mathrm{~T}^{1} a \mathrm{~T}^{1} \mathrm{~T}^{1} a \mathrm{~T}^{1} \mathrm{~T}^{1} \subseteq \mathrm{A}$.

Now $\langle a\rangle^{3}=\left(\mathrm{T}^{1} \mathrm{~T}^{1} a \mathrm{~T}^{1} \mathrm{~T}^{1}\right)\left(\mathrm{T}^{1} \mathrm{~T}^{1} a \mathrm{~T}^{1} \mathrm{~T}^{1}\right)\left(\mathrm{T}^{1} \mathrm{~T}^{1} a \mathrm{~T}^{1} \mathrm{~T}^{1}\right) \subseteq \mathrm{T}^{1} \mathrm{~T}^{1} a \mathrm{~T}^{1} \mathrm{~T}^{1} a \mathrm{~T}^{1} \mathrm{~T}^{1} a \mathrm{~T}^{1} \mathrm{~T}^{1} \subseteq \mathrm{A} \Rightarrow a \in \mathrm{A}$.

(iii) $\Rightarrow$ (i): Suppose that $a \in \mathrm{T} ; \mathrm{T}^{1} \mathrm{~T}^{1} a \mathrm{~T}^{1} \mathrm{~T}^{1} a \mathrm{~T}^{1} \mathrm{~T}^{1} a \mathrm{~T}^{1} \mathrm{~T}^{1} \subseteq \mathrm{A} \Rightarrow a \in \mathrm{A}$.

Let $\mathrm{X}$ be the an ideals of $\mathrm{T}$ and $\mathrm{X}^{3} \subseteq \mathrm{A}$.

Suppose if possible $\mathrm{X} \nsubseteq \mathrm{A}$.

$\mathrm{X} \nsubseteq \mathrm{A}$ there exists $a$ such that $a \in \mathrm{X}$ and $a \notin \mathrm{A} . a \in \mathrm{X} \Rightarrow a^{3} \in \mathrm{X}^{3} \subseteq \mathrm{A}$.

Now $\mathrm{T}^{1} \mathrm{~T}^{1} a \mathrm{~T}^{1} \mathrm{~T}^{1} a \mathrm{~T}^{1} \mathrm{~T}^{1} a \mathrm{~T}^{1} \mathrm{~T}^{1} \subseteq \mathrm{X}^{3} \subseteq \mathrm{A} \Rightarrow a \in \mathrm{A}$. It is a contradiction.

Therefore $\mathrm{X} \subseteq \mathrm{A}$ and hence $\mathrm{A}$ is a semiprime ideal of $\mathrm{T}$. 
THEOREM 4.15 : Every completely semiprime ideal of a ternary semigroup $\mathrm{T}$ is a semiprime ideal of $\mathrm{T}$. Proof: Suppose that A is a completely semiprime ideal of a ternary semigroup T.

Let $a \in \mathrm{T}$ and $\langle a\rangle^{n} \subseteq \mathrm{A}$ for some odd natural number $n$.

Now $a a a . . . . a(n$ odd terms $) \in\left\langle a^{n}\right\rangle \subseteq\langle a\rangle^{n} \subseteq \mathrm{A} \Rightarrow a^{n} \in \mathrm{A} \Rightarrow a \in \mathrm{A} \Rightarrow\langle a\rangle \subseteq \mathrm{A}$.

Therefore $\mathrm{A}$ is a semiprime ideal of $\mathrm{T}$.

THEOREM 4.16 : Let $\mathbf{T}$ be a commutative ternary semigroup. An ideal A of $\mathbf{T}$ is completely semiprime if and only if it is semiprime.

Proof: Suppose that A is a completely semiprime ideal of T. By theorem 4.14, A is a semiprime ideal of T.

Conversely suppose that $\mathrm{A}$ is a semiprime ideal of $\mathrm{T}$.

Let $x \in \mathrm{T}$ and $x^{n} \in \mathrm{A}$ for some odd natural number $n$.

Now $x^{n} \in \mathrm{A} \Rightarrow\left\langle x>^{n} \subseteq \mathrm{A} \Rightarrow\langle x\rangle \subseteq \mathrm{A} \Rightarrow x \in \mathrm{A}\right.$. Since $\mathrm{A}$ is semiprime.

Therefore $\mathrm{A}$ is a completely semiprime ideal of $\mathrm{T}$.

THEOREM 4.17 : The nonempty intersection of any family of prime ideals of a ternary semigroup $T$ is a semiprime ideal of $T$.

Proof : Let $\left\{A_{\alpha}\right\}_{\alpha \in \Delta}$ be a family of prime ideals of $\mathrm{T}$ such that $\bigcap_{\alpha \in \Delta} A_{\alpha} \neq \varnothing$. It is clear that $\bigcap_{\alpha \in \Delta} A_{\alpha}$ is an ideal. Let $a \in T,\langle a\rangle^{3} \subseteq \bigcap_{\alpha \in \Delta} A_{\alpha}$ then $\langle a\rangle^{3} \subseteq A_{\alpha}$ for all $\alpha \in \Delta$.

Since $A_{\alpha}$ is a prime, $<a>\subseteq A_{\alpha}$ for all $\alpha \in \Delta$ and hence $a \in A_{\alpha}$ for all $\alpha \in \Delta$.

So $a \in \bigcap_{\alpha \in \Delta} A_{\alpha}$. Therefore $\bigcap_{\alpha \in \Delta} A_{\alpha}$ is a semiprime ideal of $\mathrm{T}$.

DEFINITION 4.18 : A non-empty subset $A$ of a ternary semigroup $T$ is said to be an $n$-system provided for any $a \in \mathrm{A}$ implies that $\mathrm{T}^{1} \mathrm{~T}^{1} a \mathrm{~T}^{1} \mathrm{~T}^{1} a \mathrm{~T}^{1} \mathrm{~T}^{1} a \mathrm{~T}^{1} \mathrm{~T}^{1} \cap \mathrm{A} \neq \emptyset$.

THEOREM 4.19 : Every $\boldsymbol{m}$-system in a ternary semigroup $\mathrm{T}$ is an $\boldsymbol{n}$-system.

Proof : Let A be $m$-system of a ternary semigroup T. Let $a \in A$. Since A is $m$-system, $a \in \mathrm{A}, \mathrm{T}^{1} \mathrm{~T}^{1} a \mathrm{~T}^{1} \mathrm{~T}^{1} a \mathrm{~T}^{1} \mathrm{~T}^{1} a \mathrm{~T}^{1} \mathrm{~T}^{1} \cap \mathrm{A} \neq \emptyset$. Therefore $A$ is an $n$-system of $\mathrm{T}$.

THEOREM 4.20 : An ideal $Q$ of a ternary semigroup $T$ is a semiprime ideal if and only if T/Q is an $n$ system of $T$ (or) empty.

Proof: Suppose that $\mathrm{A}$ is a semiprime ideal of a ternary semigroup $\mathrm{T}$ and $\mathrm{T} \backslash \mathrm{A} \neq \varnothing$.

Let $a \in \mathrm{T} \backslash \mathrm{A}$. Then $a \notin \mathrm{A}$.

Suppose if possible $\mathrm{T}^{1} \mathrm{~T}^{1} a \mathrm{~T}^{1} \mathrm{~T}^{1} a \mathrm{~T}^{1} \mathrm{~T}^{1} a \mathrm{~T}^{1} \mathrm{~T}^{1} \cap \mathrm{T} \backslash \mathrm{A}=\emptyset$.

$\mathrm{T}^{1} \mathrm{~T}^{1} a \mathrm{~T}^{1} \mathrm{~T}^{1} a \mathrm{~T}^{1} \mathrm{~T}^{1} a \mathrm{~T}^{1} \mathrm{~T}^{1} \cap \mathrm{T} \backslash \mathrm{A}=\emptyset \Rightarrow \mathrm{T}^{1} \mathrm{~T}^{1} a \mathrm{~T}^{1} \mathrm{~T}^{1} a \mathrm{~T}^{1} \mathrm{~T}^{1} a \mathrm{~T}^{1} \mathrm{~T}^{1} \subseteq \mathrm{A}$.

Since $\mathrm{A}$ is semiprime, either $a \in \mathrm{A}$.

It is a contradiction. Therefore $\mathrm{T}^{1} \mathrm{~T}^{1} a \mathrm{~T}^{1} \mathrm{~T}^{1} a \mathrm{~T}^{1} \mathrm{~T}^{1} a \mathrm{~T}^{1} \mathrm{~T}^{1} \cap \mathrm{T} \backslash \mathrm{A} \neq \emptyset$.

Hence T\A is an $n$-system.

Conversely suppose that $\mathrm{T} \backslash \mathrm{A}$ is either an $n$-system or $\mathrm{T} \backslash \mathrm{A}=\varnothing$.

If $\mathrm{T} \backslash \mathrm{A}=\varnothing$ then $\mathrm{T}=\mathrm{A}$ and hence $\mathrm{A}$ is a semiprime ideal.

Assume that $\mathrm{T} \backslash \mathrm{A}$ is an $n$-system of $\mathrm{T}$. Let $a \in \mathrm{T}$ and $\langle a\rangle \subseteq \mathrm{A}$.

Let $a \in \mathrm{T} \backslash \mathrm{A}$, T\A is an $n$-system of $\mathrm{T} \Rightarrow \mathrm{T}^{1} \mathrm{~T}^{1} a \mathrm{~T}^{1} \mathrm{~T}^{1} a \mathrm{~T}^{1} \mathrm{~T}^{1} a \mathrm{~T}^{1} \mathrm{~T}^{1} \cap \mathrm{T} \backslash \mathrm{A} \neq \emptyset$.

Suppose if possible $a \notin \mathrm{A}$. Then $a \in \mathrm{T} \backslash \mathrm{A}$. Since $\mathrm{T} \backslash \mathrm{A}$ is an $m$-system.

Then $\mathrm{T}^{1} \mathrm{~T}^{1} a \mathrm{~T}^{1} \mathrm{~T}^{1} a \mathrm{~T}^{1} \mathrm{~T}^{1} a \mathrm{~T}^{1} \mathrm{~T}^{1} \subseteq \mathrm{T} \backslash \mathrm{A} \Rightarrow \mathrm{T}^{1} \mathrm{~T}^{1} a \mathrm{~T}^{1} \mathrm{~T}^{1} a \mathrm{~T}^{1} \mathrm{~T}^{1} a \mathrm{~T}^{1} \mathrm{~T}^{1} \nsubseteq \mathrm{A} \Rightarrow\langle a\rangle \nsubseteq \mathrm{A}$.

It is a contradiction. Therefore $a \in \mathrm{A}$. Hence $\mathrm{A}$ is a semiprime ideal of $\mathrm{T}$.

THEOREM 4.21 : If $N$ is an $n$-system in a ternary semigroup $T$ and $a \in N$, then there exist an $m$-system $M$ in $T$ such that $a \in M$ and $M \subseteq N$.

Proof: We construct a subset $\mathrm{M}$ of $\mathrm{N}$ as follows:

Define $a_{1}=a$, Since $a_{1} \in \mathrm{N}$ and $\mathrm{N}$ is an $n$-system, $\left(\mathrm{T}^{1} \mathrm{~T}^{1} a_{1} \mathrm{~T}^{1} \mathrm{~T}^{1} a_{1} \mathrm{~T}^{1} \mathrm{~T}^{1} a_{1} \mathrm{~T}^{1} \mathrm{~T}^{1}\right) \cap \mathrm{N} \neq \varnothing$.

Let $a_{2} \in\left(\mathrm{T}^{1} \mathrm{~T}^{1} a_{1} \mathrm{~T}^{1} \mathrm{~T}^{1} a_{1} \mathrm{~T}^{1} \mathrm{~T}^{1} a_{1} \mathrm{~T}^{1} \mathrm{~T}^{1}\right) \cap \mathrm{N}$. Since $a_{2} \in \mathrm{N}$ and $\mathrm{N}$ is an $n$-system, $\left(\mathrm{T}^{1} \mathrm{~T}^{1} a_{2} \mathrm{~T}^{1} \mathrm{~T}^{1} a_{2} \mathrm{~T}^{1} \mathrm{~T}^{1} a_{2} \mathrm{~T}^{1} \mathrm{~T}^{1}\right) \cap \mathrm{N}$ $\neq \varnothing$ and so on.

In general, if $a_{i}$ has been defined with $a_{i} \in \mathrm{N}$, choose $a_{i+1}$ as an element of $\left(\mathrm{T}^{1} \mathrm{~T}^{1} a_{2} \mathrm{~T}^{1} \mathrm{~T}^{1} a_{2} \mathrm{~T}^{1} \mathrm{~T}^{1} a_{2} \mathrm{~T}^{1} \mathrm{~T}^{1}\right) \cap \mathrm{N}$. Let $\mathrm{M}=\left\{a_{1}, a_{2} \ldots . a_{i}, a_{i+1} \ldots.\right\}$. Now $a \in \mathrm{M}$ and $\mathrm{M} \subseteq \mathrm{N}$. 
We now show that $\mathrm{M}$ is an $m$-system.

Let $a_{i}, a_{j}, a_{k} \in \mathrm{M}$ (for $i \leq j \leq k$ ).

Then $a_{k+1} \in \mathrm{T}^{1} \mathrm{~T}^{1} a_{k} \mathrm{~T}^{1} \mathrm{~T}^{1} a_{k} \mathrm{~T}^{1} \mathrm{~T}^{1} a_{k} \mathrm{~T}^{1} \mathrm{~T}^{1} \subseteq \mathrm{T}^{1} \mathrm{~T}^{1} a_{j} \mathrm{~T}^{1} \mathrm{~T}^{1} a_{j} \mathrm{~T}^{1} \mathrm{~T}^{1} a_{j} \mathrm{~T}^{1} \mathrm{~T}^{1}$

$\subseteq \mathrm{T}^{1} \mathrm{~T}^{1} a_{i} \mathrm{~T}^{1} \mathrm{~T}^{1} a_{i} \mathrm{~T}^{1} \mathrm{~T}^{1} a_{i} \mathrm{~T}^{1} \mathrm{~T}^{1} \subseteq \mathrm{T}^{1} \mathrm{~T}^{1} a_{i} \mathrm{~T}^{1} \mathrm{~T}^{1} a_{j} \mathrm{~T}^{1} \mathrm{~T}^{1} a_{k} \mathrm{~T}^{1} \mathrm{~T}^{1}$

$\Rightarrow a_{k+1}=\mathrm{T}^{1} \mathrm{~T}^{1} a_{i} \mathrm{~T}^{1} \mathrm{~T}^{1} a_{j} \mathrm{~T}^{1} \mathrm{~T}^{1} a_{k} \mathrm{~T}^{1} \mathrm{~T}^{1}$. But $a_{k+1} \in \mathrm{M}$, so $a_{k+1}=\mathrm{T}^{1} \mathrm{~T}^{1} a_{i} \mathrm{~T}^{1} \mathrm{~T}^{1} a_{j} \mathrm{~T}^{1} \mathrm{~T}^{1} a_{k} \mathrm{~T}^{1} \mathrm{~T}^{1} \cap \mathrm{M}$,

Therefore $\mathrm{M}$ is an $m$-system.

\section{Prime Radical And Completely Prime Radical}

NOTATION 5.1 : If $\mathrm{A}$ is an ideal of a ternary semigroup T, then we associate the following four types of sets.

$A_{1}=$ The intersection of all completely prime ideals of $\mathrm{T}$ containing A.

$A_{2}=\left\{x \in T: x^{n} \in A\right.$ for some odd natural numbers $\left.n\right\}$

$A_{3}=$ The intersection of all prime ideals of $\mathrm{T}$ containing A.

$A_{4}=\left\{x \in T:\langle x\rangle^{n} \subseteq\right.$ A for some odd natural number $\left.n\right\}$

THEOREM 5.2 : If $\mathbf{A}$ is an ideal of a ternary semigroup $\mathbf{T}$, then $\mathrm{A} \subseteq A_{4} \subseteq A_{3} \subseteq A_{2} \subseteq A_{1}$.

Proof: i) $\mathrm{A} \subseteq A_{4}$ : Let $x \in \mathrm{A}$. Then $\langle x\rangle \subseteq \mathrm{A}$ and hence $x \in A_{4}$

Therefore $\mathrm{A} \subseteq A_{4}$

ii) $A_{4} \subseteq A_{3}$ : Let $x \in A_{4}$. Then $\langle x\rangle^{n} \subseteq \mathrm{A}$ for some odd natural number $n$.

Let $\mathrm{P}$ be any prime ideal of $\mathrm{T}$ containing $\mathrm{A}$.

Then $\langle x\rangle^{n} \subseteq$ A for some odd natural number $n \Rightarrow\langle x\rangle^{n} \subseteq \mathrm{P}$.

Since $\mathrm{P}$ is prime,$\langle x\rangle \subseteq \mathrm{P}$ and hence $x \in \mathrm{P}$.

Since this is true for all prime ideals of $\mathrm{P}$ containing $\mathrm{A}, x \in A_{3}$. Therefore $A_{4} \subseteq A_{3}$

iii) $A_{3} \subseteq A_{2}$ : Let $x \in A_{3}$. Suppose if possible $x \notin A_{2}$.

Then $x^{n} \notin \mathrm{A}$ for all odd natural number $n$.

Consider $\mathrm{Q}=\bigcup x^{n}$ for all odd natural number $n$, and $x \in \mathrm{T}$.

Let $a, b, c \in \mathrm{Q}$. Then $a=(x)^{r}, b=(x)^{s}, c=(x)^{t}$ for some odd natural numbers $r, s, t$.

Therefore $a b c=(x)^{r}(x)^{s}(x)^{t}=x^{r+s+t} \in Q$ and hence $\mathrm{Q}$ is a subsemigroup of $\mathrm{T}$.

By theorem 3.5, $\mathrm{P}=\mathrm{T} \backslash \mathrm{Q}$ is a completely prime ideal of $\mathrm{T}$ and $x \notin P$.

By theorem 3.9, $\mathrm{P}$ is a prime ideal of $\mathrm{T}$ and $x \notin P$. Therefore $x \notin A_{3}$. It is a contradiction.

Therefore $x \in A_{2}$ and hence $A_{3} \subseteq A_{2}$.

iv) $A_{2} \subseteq A_{1}:$ Let $x \in A_{2}$. Now $\mathrm{x} \in A_{2} \Rightarrow x^{n} \in \mathrm{A}$ for some odd natural number $n$.

Let $\mathrm{P}$ be any completely prime ideal of $\mathrm{T}$ containing $\mathrm{A}$.

Then $x^{n} \in \mathrm{A} \subseteq \mathrm{P} \Rightarrow x^{n} \in \mathrm{P} \Rightarrow x \in \mathrm{P}$. Therefore $x \in A_{1}$. Therefore $A_{2} \subseteq A_{1}$.

Hence $\mathrm{A} \subseteq A_{4} \subseteq A_{3} \subseteq A_{2} \subseteq A_{1}$.

THEOREM 5.3 : $\mathbf{A}$ is an ideal of a commutative ternary semigroup $\mathbf{T}$, then $A_{1}=A_{2}=A_{3}=A_{4}$

Proof: By theorem5.2, $\mathrm{A} \subseteq A_{4} \subseteq A_{3} \subseteq A_{2} \subseteq A_{1}$. By theorem 3.10, in a commutative ternary semigroup $\mathrm{T}$, an ideal $\mathrm{A}$ is a prime ideal if $\mathrm{A}$ is completely prime ideal.

So $A_{1}=A_{3}$. By theorem 4.16, in a commutative ternary semigroup $\mathrm{T}$ an ideal $\mathrm{A}$ is semiprime if and only if $\mathrm{A}$ is completely semiprime ideal.

So $A_{4}=A_{2}$ and hence $A_{1}=A_{2}=A_{3}=A_{4}$.

NOTE 5.4 : In an arbitrary ternary semigroup $A_{1} \neq A_{2} \neq A_{3} \neq A_{4}$.

EXAMPLE 5.5 : Let $\mathrm{T}$ be the free ternary semigroup generated by $a, b, c$.

It is clear that $\mathrm{A}=\mathrm{T} a^{3} \mathrm{~T}$ is an ideal of $\mathrm{T}$. Since $a^{5} \in \mathrm{T} a^{3} \mathrm{~T}$, we have $\mathrm{a} \in A_{2}$. 
Evidently $(a b c)^{n} \notin \mathrm{T} a^{3} \mathrm{~T}$ for all odd natural numbers $n$ and thus abc $\notin A_{2}$.

Thus $A_{2}$ is not an ideal of T. Therefore $A_{1} \neq A_{2}$ and $A_{2} \neq A_{3}$.

DEFINITION 5.6 : If $\mathrm{A}$ is an ideal of a ternary semigroup $\mathrm{T}$, then the intersection of all prime ideals of $\mathrm{T}$ containing A is called prime radical or simply radical of $\mathrm{A}$ and it is denoted by $\sqrt{A}$ or $\mathrm{rad} \mathrm{A}$.

DEFINITION 5.7: If $A$ is an ideal of a ternary semigroup $T$, then the intersection of all completely prime ideals of $\mathrm{T}$ containing $\mathrm{A}$ is called completely prime radical or simply complete radical of $\mathrm{A}$ and it is denoted by c.rad A.

NOTE 5.8: If $\mathrm{A}$ is an ideal of a ternary semigroup $\mathrm{T}$, then $\operatorname{rad} \mathrm{A}=A_{3}$ and $\operatorname{crad} \mathrm{A}=A_{1}$.

THEOREM 5.9: If $a \in \sqrt{A}$, then there exist a positive integer $\boldsymbol{n}$ such that $a^{n} \in \mathrm{A}$ for some odd natural number $n \in \mathbf{N}$.

Proof: By theorem 5.2, $A_{3} \subseteq A_{2}$ and hence $a \in \sqrt{A}=A_{3} \subseteq A_{2}$.

Therefore $a^{n} \in \mathbf{A}$ for some odd natural number $n \in \mathrm{N}$.

THEOREM 5.10 : If $A$ is an ideal of a commutative ternary semigroup $T$, then $\operatorname{rad} \mathrm{A}=$ c.rad $\mathrm{A}$.

proof : By theorem 5.3, rad $\mathrm{A}=$ c.rad $\mathrm{A}$.

THEOREM 5.11 : If $\mathrm{A}$ is an ideal of a ternary semigroup $\mathrm{T}$ then $c . r a d \mathrm{~A}$ is a completely semiprime ideal of $\mathbf{T}$.

proof : By theorem 4.6, c.rad A is a completely semiprime ideal of $\mathrm{T}$.

THEOREM 5.12 : If $A, B$ and $C$ are any three ideals of a ternary semigroup $T$, then

i) $\mathbf{A} \subseteq \mathbf{B} \Rightarrow \sqrt{A} \subseteq \sqrt{B}$

ii) if $\mathbf{A} \square \mathbf{B} \square \mathbf{C} \emptyset$ then $\sqrt{A B C}=\sqrt{A \cap B \cap C}=\sqrt{A} \cap \sqrt{B} \cap \sqrt{C}$

iii) $\sqrt{\sqrt{A}}=\sqrt{A}$.

proof : i) Suppose that $\mathrm{A} \subseteq \mathrm{B}$. If $\mathrm{P}$ is a prime ideal containing $\mathrm{B}$ then $\mathrm{P}$ is a prime ideal containing $\mathrm{A}$. Therefore $\sqrt{A} \subseteq \sqrt{B}$

ii) Let $\mathrm{P}$ be a prime ideal containing $\mathrm{ABC}$. Then $\mathrm{ABC} \subseteq \mathrm{P} \Rightarrow \mathrm{A} \subseteq \mathrm{P}$ or $\mathrm{B} \subseteq \mathrm{P}$ or $\mathrm{C} \subseteq \mathrm{P}$

$\Rightarrow A \cap B \cap C \subseteq \mathrm{P}$. Therefore $\mathrm{P}$ is a prime ideal containing $\mathrm{A} \cap \mathrm{B} \cap \mathrm{C}$.

Therefore $\operatorname{rad}(A \cap B \cap C) \subseteq \operatorname{rad}(\mathrm{ABC})$.

Now let $\mathrm{P}$ be a prime ideal containing $A \cap B \cap C$.

Then $A \cap B \cap C \subseteq \mathrm{P} \Rightarrow \mathrm{ABC} \subseteq A \cap B \cap C \subseteq \mathrm{P} \Rightarrow \mathrm{ABC} \subseteq \mathrm{P}$.

Hence $\mathrm{P}$ is a prime ideal containing $\mathrm{ABC}$. Therefore $\operatorname{rad}(\mathrm{ABC}) \subseteq \operatorname{rad}(A \cap B \cap C)$.

Therefore $\operatorname{rad}(\mathrm{ABC})=\operatorname{rad}(A \cap B \cap C)$.

Since $A \cap B \cap C \neq \varnothing$, it is clear that $\mathrm{A} \cap \mathrm{B}$ is an ideal in T. Let $x \in \sqrt{A \cap B \cap C}$.

Then there exists a odd natural number $n \in \mathrm{N}$ such that $x^{n} \in A \cap B \cap C$.

Therefore $x^{n} \in \mathrm{A}, x^{n} \in \mathrm{B}$ and $x^{n} \in \mathrm{C}$. It follows that $x \in \sqrt{A}, x \in \sqrt{B}$ and $x \in \sqrt{C}$. Therefore $x$ $\in \sqrt{A} \cap \sqrt{B} \cap \sqrt{C}$.

Consequently, $x \in \sqrt{A} \cap \sqrt{B} \cap \sqrt{C}$ implies that there exists odd natural numbers $n, m, p \in \mathrm{N}$ such that $x^{n} \in \mathrm{A}, x^{m} \in \mathrm{B}$ and $x^{p} \in \mathrm{C}$. Clearly, $x^{n m p} \in \mathrm{A} \cap \mathrm{B} \cap \mathrm{C}$.

Thus $x \in \sqrt{A \cap B \cap C}$. Therefore if $\mathrm{A} \cap \mathrm{B} \cap \mathrm{C} \neq \emptyset$ then $\sqrt{A \cap B \cap C}=\sqrt{A} \cap \sqrt{B} \cap \sqrt{C}$.

iii) $\sqrt{A}=$ The intersection of all prime ideals of $\mathrm{T}$ containing A.

Now $\sqrt{\sqrt{A}}=$ The intersection of all prime ideals of $\mathrm{T}$ containing $\sqrt{A}$.

$=$ The intersection of all prime ideals of $\mathrm{T}$ containing $\mathrm{A}=\sqrt{A}$

Therefore $\sqrt{\sqrt{A}}=\sqrt{A}$.

THEOREM 5.13 : If $\mathbf{A}$ is an ideal of a ternary semigroup $\mathbf{T}$ then $\sqrt{A}$ is a semiprime ideal of $\mathbf{T}$. 
proof : By theorem 4.17, $\sqrt{A}$ is a semiprime ideal of $\mathrm{T}$.

THEOREM 5.14 : An ideal $Q$ of ternary semigroup $\mathbf{T}$ is a semiprime ideal of $\mathbf{T}$ if and only if $\sqrt{Q}=\mathbf{Q}$.

Proof: Suppose that $\mathrm{Q}$ is a semiprime ideal. Clearly $\mathrm{Q} \subseteq \sqrt{ } \mathrm{Q}$.

Suppose if possible $\sqrt{ } \mathrm{Q} \nsubseteq \mathrm{Q}$.

Let $a \in \sqrt{ } \mathrm{Q}$ and $a \notin \mathrm{Q}$. Now $a \notin \mathrm{Q} \Rightarrow a \in \mathrm{S} \backslash \mathrm{Q}$ and $\mathrm{Q}$ is semiprime. By theorem 4.20,

$\mathrm{SIQ}$ is an $n$-system. By theorem 4.21, there exists an $m$-system M such that $a \in \mathrm{M} \subseteq \mathrm{S} \backslash \mathrm{Q}$.

$\mathrm{Q} \subseteq \mathrm{S} \backslash \mathrm{M}$ and now SLM is a prime ideal of $\mathrm{S}, a \notin \mathrm{SLM}$. It is a contradiction.

Therefore $\sqrt{ } \mathrm{Q} \subseteq \mathrm{Q}$. Hence $\sqrt{\mathrm{Q}}=\mathrm{Q}$.

Conversely suppose that $\mathrm{Q}$ is an ideal of $\mathrm{S}$ such that $\sqrt{ } \mathrm{Q}=\mathrm{Q}$.

By corollary 5.13, $\sqrt{ } \mathrm{Q}$ is a semiprime ideal of $\mathrm{S}$. Therefore $\mathrm{Q}$ is semiprime.

COROLLARY 5.15 : An ideal $Q$ of a ternary semigroup $T$ is a semiprime ideal if and only if $Q$ is the intersection of all prime ideal of $S$ contains $Q$.

Proof: By theorem 5.14., Q is semiprime iff $\mathrm{Q}$ is the intersection of all prime ideals of $\mathrm{T}$ contains $\mathrm{Q}$.

COROLLARY 5.16 : If $A$ is an ideal of a ternary semi group $T$, then $A$ is the smallest semiprime ideal of T containing A.

Proof: We have that $\sqrt{ } \mathrm{A}$ is the intersection of all prime ideals containing $\mathrm{A}$ in $\mathrm{T}$.

Since intersection of prime ideals is semiprime, we have $\sqrt{ } \mathrm{A}$ is semiprime.

Further, let $\mathrm{Q}$ be any semiprime ideal containing $\mathrm{A}$, i.e. $\mathrm{A} \subseteq \mathrm{Q}$. So $\sqrt{\mathrm{A}} \subseteq \sqrt{\mathrm{Q}}$.

Since $\mathrm{Q}$ is semiprime, By theorem 5.14, $\sqrt{ } \mathrm{Q}=\mathrm{Q}$. Therefore $\sqrt{\mathrm{A}} \subseteq \mathrm{Q}$.

Hence $\sqrt{ } \mathrm{A}$ is the smallest semiprime ideal of $\mathrm{S}$ containing $\mathrm{A}$.

THEOREM 5.16 : If $\mathbf{P}$ is a prime ideal of a ternary semigroup T, then $\sqrt{(P)^{n}}=\boldsymbol{P}$ for all odd natural numbers $n \in \mathbf{N}$.

Proof: We use induction on $n$ to prove $\sqrt{P^{n}}=P$.

First we prove that $\sqrt{P}=P$. Since $P$ is a prime ideal, $P \subseteq \sqrt{P} \subseteq P \Rightarrow \sqrt{P}=P$.

Assume that $\sqrt{P^{k}}=P$ for odd natural number k such that $1 \leq k<n$.

Now $\sqrt{P^{k+2}}=\sqrt{P^{k} . P . P}=\sqrt{P^{k}} \cap \sqrt{P} \cap \sqrt{P}=\sqrt{P} \cap \sqrt{P} \cap \sqrt{P}=\sqrt{P}=P$.

Therefore $\sqrt{P^{k+2}}=P$. By induction $\sqrt{P^{n}}=\mathrm{P}$ for all odd natural number $n \in \mathrm{N}$.

THEOREM 5.17: In a ternary semigroup $T$ with identity there is a unique maximal ideal $M$ such that $\sqrt{(M)^{n}}=\mathbf{M}$ for all odd natural numbers $n \in \mathbf{N}$.

Proof: Since T contains identity, $\mathrm{T}$ is a globally idempotent ternary semigroup.

Since $\mathrm{M}$ is a maximal ideal of $\mathrm{T}$, by theorem $3.15 \mathrm{M}$ is prime.

By theorem 5.16, $\sqrt{(M)^{n}}=\mathrm{M}$ for all odd natural numbers $n$.

Theorem 5.18: If $\mathbf{A}$ is an ideal of a ternary semigroup $\mathbf{T}$ then $\sqrt{A}=\left\{\begin{array}{lll}x \in \mathbf{T} & \text { : every }\end{array}\right.$ $m$-system of T containing $x$ meets A $\}$ i.e., $\sqrt{A}=\{x \in T: M(x) \bigcap A \neq \varnothing\}$.

Proof: Suppose that $x \in \sqrt{A}$. Let M be an m-system containing $x$.

Then $\mathrm{TLM}$ is a prime ideal of $\mathrm{T}$ and $x \notin \mathrm{T} \mathrm{M}$. If $\mathrm{M} \cap \mathrm{A}=\varnothing$ then $\mathrm{A} \subseteq \mathrm{T} \mathrm{M}$.

Since TLM is a prime ideal containing $\mathrm{A}, \sqrt{A} \subseteq \mathrm{T} M \mathrm{M}$ and hence $x \in \mathrm{T} M$.

It is a contradiction. Therefore $\mathrm{M}(x) \bigcap \mathrm{A} \neq \varnothing$. Hence $x \in\{x \in T: M(x) \cap A \neq \varnothing\}$.

Conversely suppose that $x \in\{x \in T: M(x) \bigcap A \neq \varnothing\}$.

Suppose if possible $x \notin \sqrt{A}$. Then there exists a prime ideal $\mathrm{P}$ containing A such that $x \notin \mathrm{P}$.

Now $\mathrm{T} \backslash \mathrm{P}$ is an $m$-system and $x \in \mathrm{T} \backslash \mathrm{P} . \quad \mathrm{A} \subseteq \mathrm{P} \Rightarrow \mathrm{T} \backslash \mathrm{P} \cap \mathrm{A}=\varnothing \Rightarrow x \notin\{x \in T: M(x) \cap A \neq \varnothing\}$.

It is a contradiction. Therefore $x \in \sqrt{A}$. Thus $\sqrt{A}=\{x \in T: M(x) \bigcap A \neq \varnothing\}$.

\section{Conclusion}

Anjaneyulu. A initiated the study of pseudo symmetric ideals in semigroups, Madhusudhana Rao. D, Anjaneyulu. A. and Gangadhara Rao. A. initiated the the study of theory of $\Gamma$-ideals in $\Gamma$-semigroups and V. B. 
Subrahmanyeswara Rao Seetamraju, Anjaneyulu and Madhusudhana Rao initiated the study of theory of ideals in partially ordered $\Gamma$-semigroups and hence the study of ideals in semigroups, $\Gamma$-semigroups and partially ordered $\Gamma$-semigorups creates a platform for the ideals in ternary semigroups.

\section{Acknowledgements}

We thank the Department of Mathematics, VSR \& NVR College, Tenlai, A.P. India for giving their valuable support in preparing this paper.

\section{References}

[1] Anjaneyulu. A and Ramakotaiah. D., on a class of semigroups, Simon - Stivin, vol .34(1980), $241-249$.

[2] Anjaneyulu. A ., Structure and ideal theory of Duo semigroups, Semigroup forum, vol .22(1981), $237-276$.

[3] Clifford A.H and Preston G.B., The algebroic theory of semigroups, vol - I American Math. Society, Province (1961).
[4] Clifford A.H and Preston G.B., The algebroic theory of semigroups, vol - II American Math. Society, Province (1967).

[5] Dutta.T.K., Kar.S abd Maity.B.K., On Ideals of regular ternary semigroups , Internal. J. Math. Math. Sci. 18 (1993), 301-308.

[6] Hewitt. E. and Zuckerman H.S., Ternary opertions and semigroups, semigroups, Proc. Sympos. Wayne State Univ., Detroit, 1968, 33-83.

[7] Iampan . A., Lateral ideals of ternary semigroups, Ukrainian Math, Bull., 4 (2007), 323-334.

[8] Kar.S ., On ideals in ternary semigroups . Int. J. Math. Gen. Sci., 18 (2003) 3013- 3023.

[9] Kar.S and Maity.B.K., Some ideals of ternary semigroups . Analele Stintifice Ale Universitath "ALI CUZA" DIN IASI(S.N) Mathematica, Tumul LVII. 2011-12.

[10] Kasner . E., An extension of the group concept, Bull. Amer. Math. Society, 10 (1904), 290-291.

[11] Kim Ki.H and Roush F.W., Ternary semigroup on each pair of factors, Simon Stevin 34(1980),No.2,63-74.

[12] Kuroki. N., Rough ideals in semigroups, Information sciences, Vol.100 (1997), 139-163.

[13] Lehmer . D.H., A ternary analave of abelian groups , Amer. J. Math., 39 (1932), 329-338

[14] Lyapin.E.S., Realisation of ternary semigroup, Russian Modern Algebra, Leningrad University, Leningrad, 1981, pp. 43-48.

[15] Petrch.M., Introduction to semigroups, Merril publishing company, Columbus, Ohio (1973).

[16] Santiago. M. L. and Bala S.S., “Ternary semigroups” Semigroups Forum,Vol. 81,no. 2, pp. 380-388, 2010.

[17] Sarala. Y, Anjaneyulu. A and Madhusudhana Rao. D., On ternary semigroups, International eJournal of Mathematics, Engineering and Technology accepted for publication.

[18] Sarala. Y, Anjaneyulu. A and Madhusudhana Rao. D., Ideals in ternary semigroups, submitted for publication in International eJournal of Mathematics, Engineering and Technology accepted for publication.

[19] Shabir.M and Basher.S., Prime Ideals in ternary semigroups Asian European J. Math. 2 (2009), 139-132.

[20] Sioson. F. M., Ideal theory in ternary semigroups , Math. Japan., 10 (1963), 63-84. 